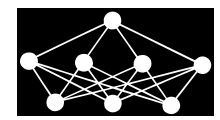

\title{
A SIMPLE ECHO ATTENUATION IN SIGNALS
}

\author{
P. Zahradnik, E.B. Aynakulov ${ }^{\dagger}$ R. Klof, B. Šimák*
}

\begin{abstract}
A simple yet powerful procedure for an echo attenuation in signals is introduced. The presented method involves no external reference signal. It is based on comb FIR filtering. To the advantages of the described method belong the simplicity and performance which are beneficial in real time implementations. For illustration, a simulation of the procedure is included. The efficiency of the presented method is demonstrated by a real time implementation on a digital signal processor.
\end{abstract}

Key words: echo attenuation, single-channel, autocorrelation, comb filter, real-time implementation, digital signal processor

Received: May 29, 2015

DOI: $10.14311 / \mathrm{NNW} .2016 .26 .017$

Revised and accepted: June 9, 2016

\section{Introduction}

An echo occurs in a signal, if, for some reason, the delayed and attenuated replica of the signal is added to the signal itself. This may be caused e.g. by a multi-path propagation, due to some unwanted feedback etc. Closely related to the echo is a reverberation which is for the lag less than $100 \mathrm{~ms}$, otherwise it is an echo. The echo attenuation is an important task in the signal processing in numerous systems. There are various applications of an echo attenuation including industrial, measuring, seismic applications, system engineering etc. We are focused here upon an echo attenuation in one-dimensional signals regardless of their origin. The signal is usually, but not necessarily, a function of a time argument. In contrast to a two-channel echo attenuation, where one of the channels is the echo-disturbed signal and the second one is a reference signal, we assume here a single channel echo attenuation. In the single-channel scenario, no external reference signal is available. A good overview in the single-channel echo attenuation can be found in $[3,9,12]$. Many adaptive methods use a finite impulse response (FIR) filter to estimate and/or cancel an echo. Numerous papers present a gradient based adaptive least mean squares (LMS) FIR filtering, see e.g. $[1,5,6]$. A common feature to all of these methods is an adaptive filtering using LMS and/or their more elaborated

\footnotetext{
*Pavel Zahradnik - Corresponding author, Radek Klof, Boris Šimák, Faculty of Electrical Eneginnering, Dept. of Telecommunication Engineering, Czech Technical University in Prague, Technicka 2, 16627 Praha 6, Czech Republic, E-mail: zahradni@fel.cvut.cz, klof@fel.cvut.cz, simak@fel.cvut.cz

$\dagger^{\dagger}$ Ermukhamed Bektaevich Aynakulov, Dept. of Energy, S. Seifullin Kazakh Agro Technical University, 62 Prospect Pobedy, Astana, Republic of Kazakhstan, E-mail: aynabekov10@mail.ru
} 
variants of LMS, e.g. the normalized LMS (NLMS). The slow convergence speed of the LMS is a well know fact. In order to speed-up the LMS convergence, the decorrelating properties of the affine projection $[2,7,8]$ and semi-orthogonal transformations [13] are used. The price to be paid for the improved performance is an increased computational complexity. Another approach, provided the feedback path is accessible, uses injection of a testing signal into the feedback path for estimating the feedback path and the FIR filter [4]. Here we are focused on a non-LMS simple single-channel echo attenuation procedure based on comb finite impulse response (FIR) filtering which is not found in references. The presented procedure does not require the access to the feedback path and is also useful in the off-line processing of echo contaminated signals. It is based on the determination of the lag of a delayed component inside the useful signal using an auto-correlation sequence and on a repetitive subtraction of the delayed components from the echo-disturbed signal which is in fact equivalent to the comb FIR filtering. The presented approach involves neither spectral transformations, nor complex arithmetics. Hence, it is well suitable in real time implementations on both prevalent implementation platforms, namely the digital signal processors (DSPs) and on field programmable gate arrays (FPGAs).

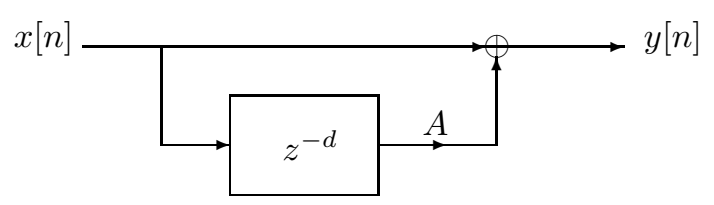

Fig. 1 Model of the echo generation.

\section{Model of the echo and of its attenuation}

Let us assume that an echo-disturbed signal $y[n]$ is composed of an input signal $x[n]$ and of the echo which is a delayed and attenuated replica of the input signal as sketched in Fig. 1. Further, let us assume that the time lag $D[\mathrm{~s}]$ of the echo is $d$ sampling periods $D=d T_{s}=d / f_{s}$ long and that the attenuation of the signal in the delayed path is $|A|<1$. Consequently we can write

$$
x[n]=y[n]-A x[n-d] .
$$

The aim of the echo attenuation is to get the approximation $x_{K}[n]$ of the echofree signal $x[n]$ from the mixture of signals $y[n]$ as precisely as possible. By analogy to Eq. (1) we get

$$
x[n-k d]=y[n-k d]-A x[n-(k+1) d], k \in \mathcal{Z} .
$$

From Eqs. (1)-(2) we can express the relation between the echo-disturbed signal $y[n]$, the echo-free signal $x[n]$ and the echo-attenuated signal $x_{K}[n]$ as follows 


$$
\begin{aligned}
x[n]= & y[n]-A x[n-d] \\
= & y[n]-A y[n-d]+A^{2} x[n-2 d] \\
= & y[n]-A y[n-d]+A^{2} y[n-2 d]-A^{3} x[n-3 d]+\ldots \\
= & \sum_{k=0}^{\infty}(-1)^{k} A^{k} y[n-k d]+(-1)^{k+1} A^{k+1} x[n-(k+1) d] \\
= & \sum_{k=0}^{K-1}(-1)^{k} A^{k} y[n-k d] \\
& \quad+\sum_{k=K}^{\infty}(-1)^{k} A^{k} y[n-k d]+(-1)^{k+1} A^{k+1} x[n-(k+1) d] \\
= & x_{K}[n]+x_{r_{K}}[n] .
\end{aligned}
$$

Eq. (3) recovers the echo-free signal $x[n]$ in an infinite number of $K \rightarrow \infty$ steps. For practical implementation reasons, we limit the summing in Eq. (3) to a finite number of $K \ll \infty$ steps. Consequently, we get the signal denoted $x_{K}[n]$ which is an approximation of the echo-free signal $x[n]$ after $K$ steps and further, of course, we can evaluate the residue error $x_{r K}[n]$ after $K$ steps Eq. (3). The corresponding signal scheme of the echo attenuation process is shown in Fig. 2. The partial summing operation

$$
x_{K}[n]=\sum_{k=0}^{K-1}(-1)^{k} A^{k} y[n-k d]
$$

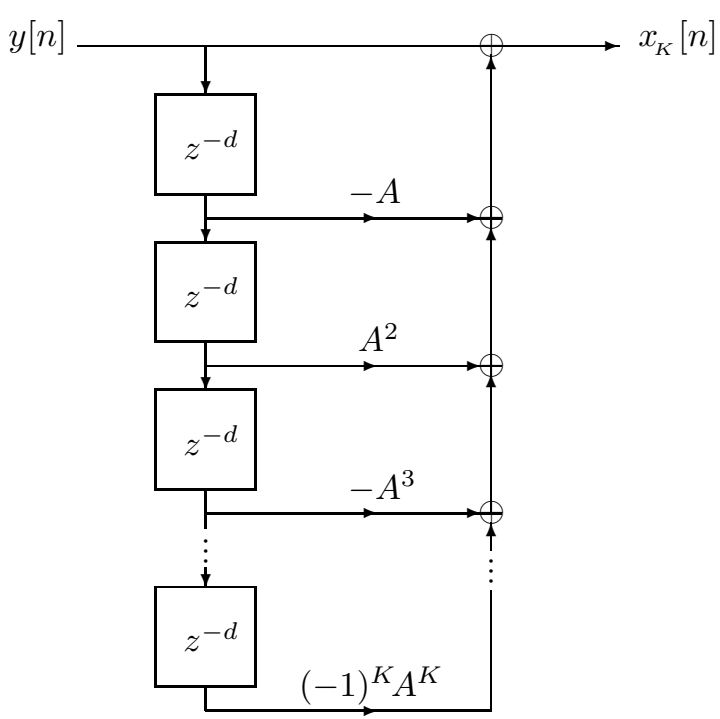

Fig. 2 Model of the echo attenuation. 
in Eq. (3) represents in fact a digital comb FIR filter with the impulse response

$$
h(m)= \begin{cases}(-1)^{m} A^{m} & \text { for } m=k d, k=0, \ldots, K-1 \\ 0 & \text { elsewhere. }\end{cases}
$$

For sake of illustration, an example of a normalized amplitude frequency response $\left|H\left(\mathrm{e}^{j \omega}\right)\right|$ corresponding to the comb FIR filter with the impulse response $h(m)$ for $A=0.75, D=100$ and $K=5$ is shown in Fig. 3 .

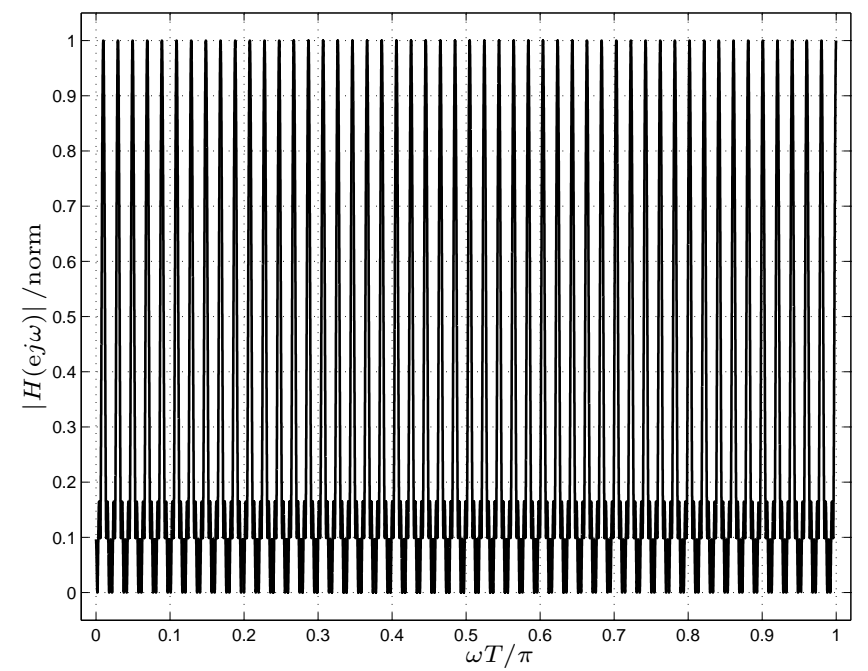

Fig. 3 Normalized amplitude frequency response $\left|H\left(\mathrm{e}^{j \omega}\right)\right|$ for $A=0.75, D=100$ and $K=5$.

\section{Echo characterization}

The echo in Eq. (1) is characterized by the time lag $D=d T_{s}[\mathrm{~s}]$ and by the attenuation value $A$. In practice, the echo attenuation procedure Eq. (3) is additionally represented by a finite number of steps $K$, see Fig. 2 as well. The lag index $d$ is evaluated reliably using the autocorrelation sequence of the echo-disturbed signal $y[n]$. Because of some practically expected maximum echo lag, we restrict, without loss of generality, the upper limit in the evaluation of the autocorrelation sequence to some reasonable value. In fact, in our real time implementation (Section 6), we evaluate the time lag $D$ within the time period $L_{1} T_{s}=0.1 \leq l T_{s} \leq L_{2} T_{s}=1[\mathrm{~s}]$, i.e. for lag indices within $0.1 f_{s} \leq l \leq f_{s}$

$$
R_{y_{\left[L_{1}, L_{2}\right]}}[l]=\sum_{n=0}^{N-l-1} y[n+l] y[n], \quad l=0.1 f_{s}, \ldots, f_{s},
$$

where $f_{s}[\mathrm{~Hz}]$ is the sampling frequency of the signal and $N$ stands for the length of a segment of the signal $y[n]$. The lag index $d$ is advantageously obtained for the maximum of the autocorrelation sequence, namely 


$$
d=l \text { for } \max _{0.1 f_{s} \leq l \leq f_{s}}\left\{\left|R_{y_{\left[L_{1}, L_{2}\right]}}[l]\right|\right\} .
$$

Further, we wish to evaluate the real attenuation value $A$. In fact, it is possible to evaluate its value for $K \rightarrow \infty$ which is not practical. In any real-time implementation, i.e. for $K \ll \infty$, we evaluate an approximation of the value $A$ which we denote $\tilde{A}$. We have developed a novel procedure for its evaluation which we present here. Let us define an auxiliary sequence

$$
f_{\varkappa}[n]=y[n]-\sum_{k=1}^{\varkappa}(-1)^{k} \tilde{A}^{k} y[n-k d]
$$

and $R_{f_{\varkappa}}[l]$ let be its autocorrelation sequence. The value $\tilde{A}$ is found for the minimum value of the autocorrelation sequence at integer multiples of the lag, i.e. for

$$
\min \left\{\sum_{k=1}^{\varkappa}\left|R_{f_{\varkappa}}[k(d+1)-1]\right|\right\} \text {. }
$$

We have found empirically that the bound $\varkappa \leq 5$ is usually sufficient in order to get the value $\tilde{A}$ within a $1 \%$ error. Further, as a rule, for $\varkappa>7$ we get no significantly more precise value of $\tilde{A}$. The determination of the required number of steps $K$ is based on the specified attenuation of the echo component $a_{e d B}$ and on the initial magnitude $A$ of the echo signal and its final value $A^{K}$ after $K$ steps, i.e.

$$
a_{e d B}=20 \log \frac{A^{K}}{A} .
$$

The required number of steps is thus

$$
K=\left\lceil\frac{a_{e d B}}{20 \log A}+1\right\rceil,
$$

where the brackets \lceil\rceil denote the up-rounding. In a real-time implementation, both values $\mathrm{d}$ and $\tilde{A}$ are updated periodically, see Section 6 .

\section{Simulation of the echo attenuation}

Let us assume an echo-free signal $x[n]$ coming from a vibration sensor sampled with sampling frequency $f_{s}=44.1 \mathrm{kHz}$. Its portion for the time period $7 \leq n T_{s} \leq 10[\mathrm{~s}]$ (Fig. 4) has the maximum value $\max \{|x[n]|\}=0.4236$ and energy $E_{x}=452.0436$. The echo free signal $x[n]$ is additively disturbed by its replica which is attenuated by the value $A=0.65$ and delayed by $d=0.45 f_{s}$ samples representing the echo time lag $D=0.45$ s, i.e. it is disturbed by the additive component $x_{d A}[n]=$ $0.65 x\left[n-0.45 f_{s}\right]$. Its maximum value is $\max \left\{\left|x_{d A}[n]\right|\right\}=0.2476$ and its energy is $E_{x_{d A}}=147.8478$. The ratio of energies is $E_{x_{d A}} / E_{x}=0.3271$. A portion of the echocorrupted signal $y[n]=x[n]+0.65 x\left[n-0.45 f_{s}\right]$ for the time period $7 \leq n T_{s} \leq 10[\mathrm{~s}]$ is shown in Fig. 5 and the difference signal $y[n]-x[n]$ is demonstrated in Fig. 6 . 


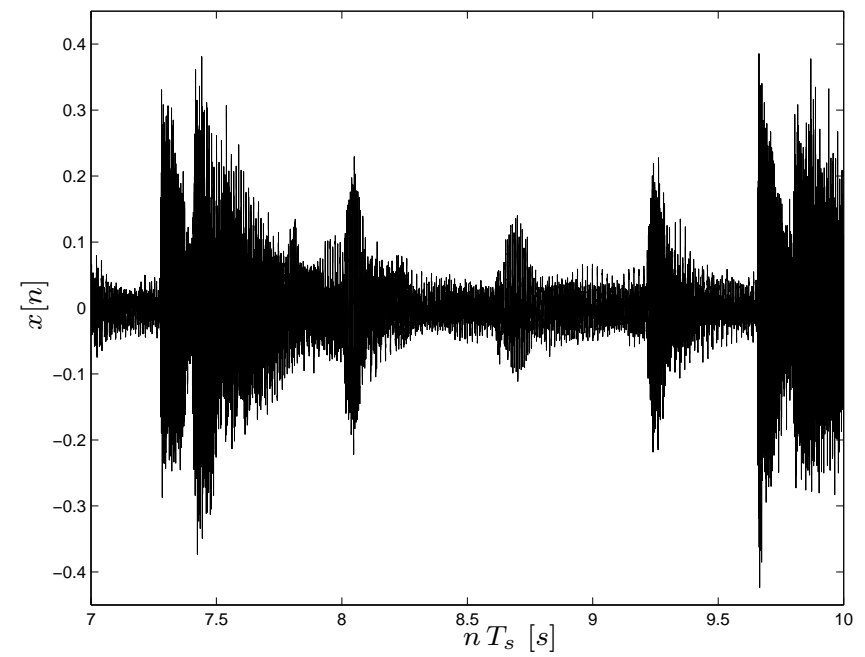

Fig. 4 Echo-free signal $x[n]$ for $7 \leq n T_{s} \leq 10[\mathrm{~s}]$.

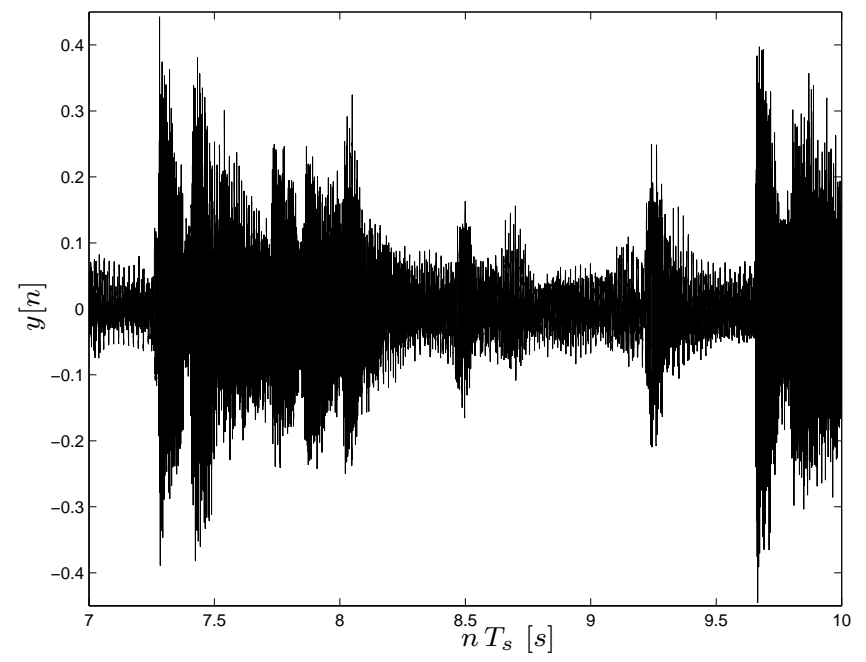

Fig. 5 Echo-disturbed signal $y[n]$ for $7 \leq n T_{s} \leq 10[\mathrm{~s}]$.

The maximum value is $\max \{|y[n]|\}=0.4450$, energy is $E_{y}=601.4510$ and the ratio of energies is $E_{y} / E_{x}=1.3305$. The autocorrelation sequence of the signal $y[n]$ for lag indices $0.1 f_{s} \leq l \leq f_{s}$ is shown in Fig. 7. The maximum value 0.4457 of the autocorrelation sequence is easily and not surprisingly found for the lag index $d=0.45 f_{s}$ (Fig. 7). i.e. for the time lag $D=0.45 \mathrm{~s}$. The attenuation value obtained using Eq. (9) is $\tilde{A}=0.6507$ for $\varkappa=5$. Its error amounts $+0.1 \%$ related to the value $A$. Now, we wish to attenuate the echo component at lest 


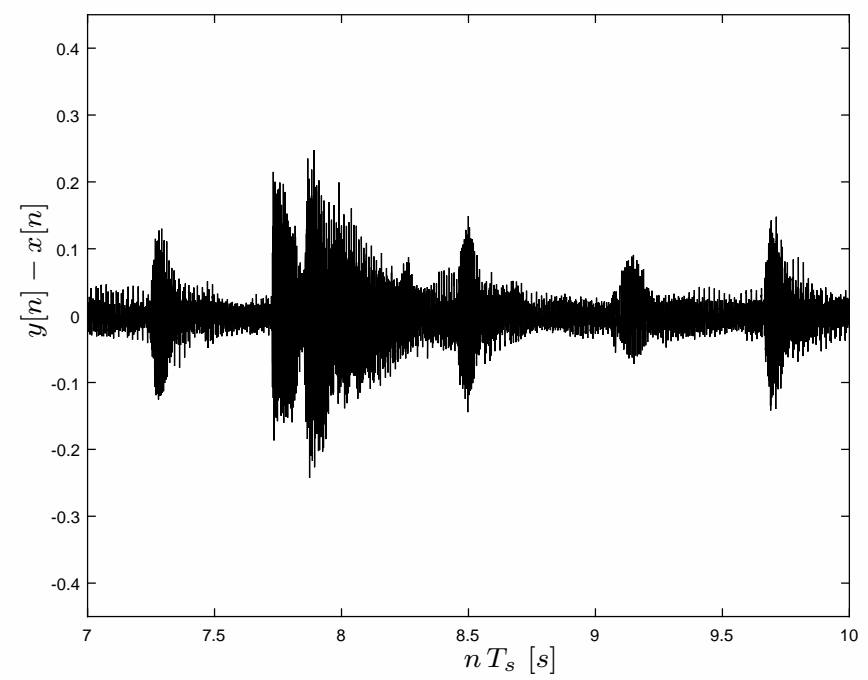

Fig. 6 Difference signal $y[n]-x[n]$ from Figs. 4 and 5.

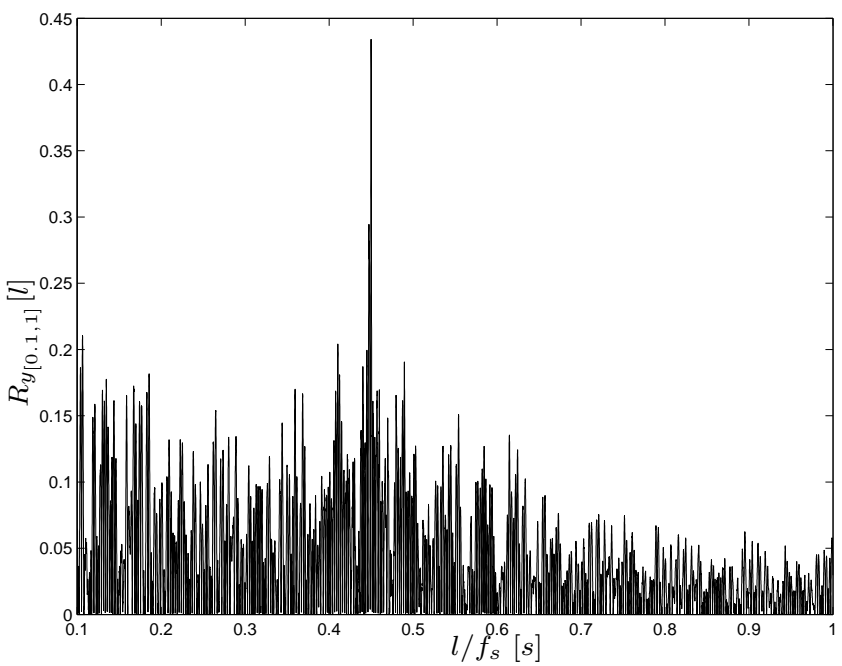

Fig. 7 Sequence $\left|R_{y_{[0.1,1]}}[l]\right|$.

hundred times, i.e. $a_{e d B} \leq-40 \mathrm{~dB}$. Using Eq. (11) we get $K=11.72 \rightarrow 12$ for $\tilde{A}=0.6507$. Finally, using Eq. (3) we get the echo-attenuated signal $x_{12}[n]$. It has the maximum value $\max \left\{\left|x_{12}[n]\right|\right\}=0.4237$, energy $E_{x_{12}}=452.0442$ and the ratio of energies is $E_{x_{12}} / E_{x}=1.00000140$. The de-echoed signal $x_{12}[n]$ (black) and the residue error signal $x_{r_{12}}[n]$ (a "white" small signal in the vicinity of the horizontal axis inside the "black" signal $\left.x_{12}[n]\right)$ are graphically compared in Fig. 8. A detailed view of the residue error signal $x_{r_{12}}[n]$ is shown in Fig. 9. The maximum value of the error signal is $\max \left\{\left|x_{r_{12}}[n]\right|\right\}=0.0026$, its energy is $E_{x_{12}}=0.0157$ a the ratio 
of energies is $E_{x_{r 12}} / E_{x}=0.000035$. The simulation demonstrates the achievement in the required echo attenuation.

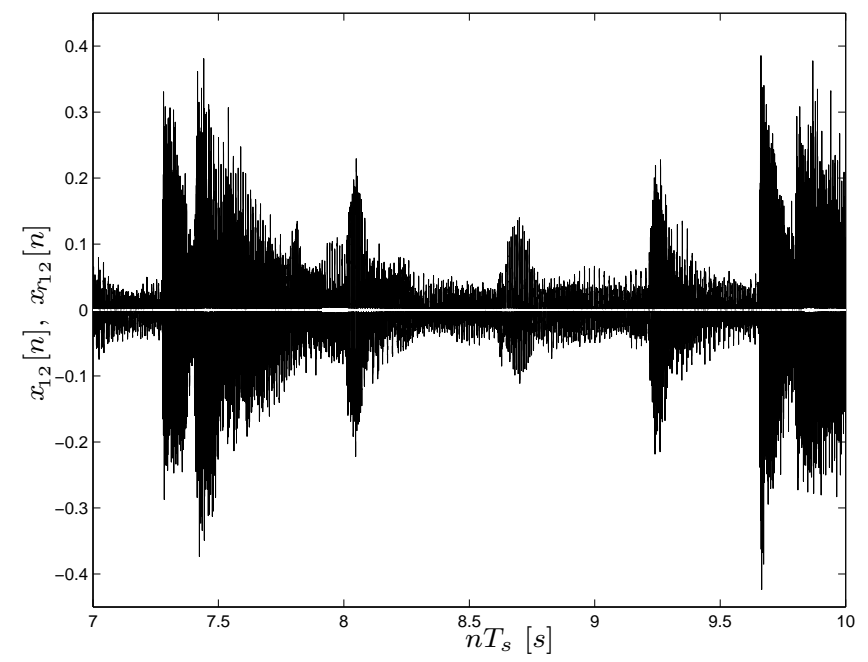

Fig. 8 De-echoed signal $x_{12}[n]$ (black) and the error signal $x_{r_{12}}[n]$ (white) near the horizontal axis.

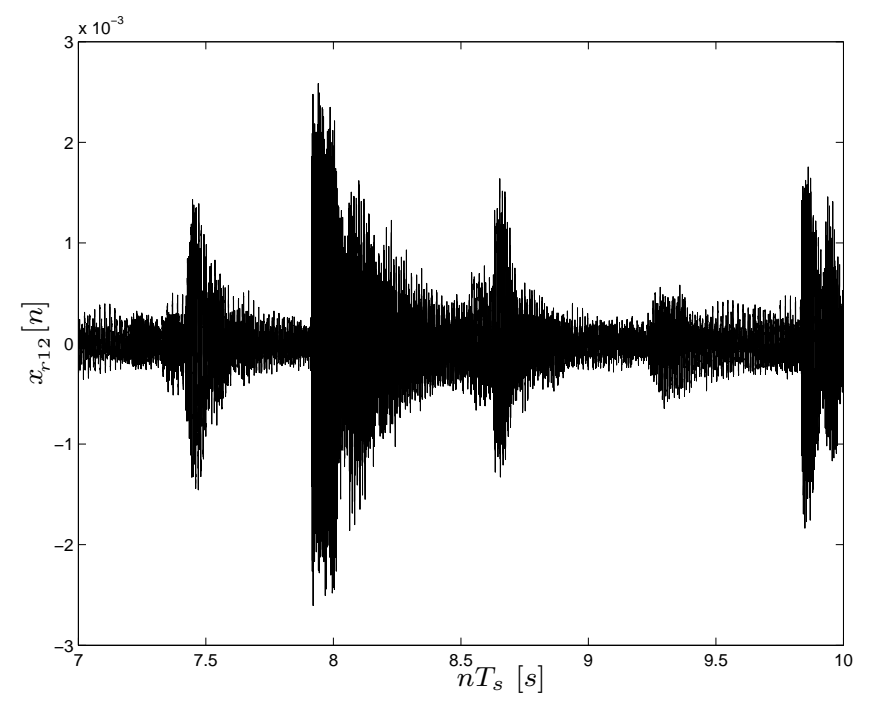

Fig. 9 A detailed view of the error signal $x_{r_{12}}[n]$. Note the vertical scale here and in Fig. 8. 


\section{Influence of Additive Noise}

The echo attenuation procedure was developed by assuming that no noise is added to the echo signal. Now, let us simulate its behaviour in the presence of an additive noise signal $x_{s}[n]$ which is added to the signal $x_{d A}[n]$. We did simulations for the Gaussian white noise where the energy of the noise signal $x_{s}[n]$ was $1 \%, 5 \%, 10 \%$, $15 \%, 20 \%, 30 \%, 40 \%$ and $50 \%$ related to the energy of the signal $x_{d A}[n]$. In all cases the lag index $d$ Eq. (7) was evaluated correctly. On the other hand the added noise affects the accuracy in the determination of the attenuation value $\tilde{A}$. Its error is summarized in Tab. I. It is apparent from Tab. I that the error in the determination of the value $\tilde{A}$ increases significantly for the relative noise energy above $30 \%$.

\begin{tabular}{rrrrrrrrr}
\hline$\frac{E_{x_{s}}}{E_{x_{d A}}}$ & 0.01 & 0.05 & 0.1 & 0.15 & 0.2 & 0.3 & 0.4 & 0.5 \\
\hline$\Delta \tilde{A}[\%]$ & 0.12 & 0.23 & 0.54 & 0.7 & 1.1 & 1.8 & 4.5 & 12.8 \\
\hline
\end{tabular}

Tab. I Error in the evaluation of $\tilde{A}$.

\section{Real-time implementation of the echo canceller}

We have implemented the proposed echo attenuation procedure on the TI OMAPL138 Development Kit (LCDK) [14]. The LCDK is based on a two-core TI OMAPL138 chip [15]. The OMAP-L138 comprises one core which is a DSP TMS320C6748 with very-long-instruction-word (VLIW) architecture consisting of eight processing units and one core ARM926EJ-S which is a RISC general purpose micro processor unit (MPU). Both cores are clocked at $456 \mathrm{MHz}$. They can work in parallel and share their data efficiently. Both cores support the floating-point and the fixedpoint arithmetics. The input signal comes in and goes out via the on-board lowpower codec TLV320AIC3106 connected to the DSP via the dedicated serial port MsASP. We use the fast on-chip 256KB SRAM solely which is sufficient for the storage of approx. $2.75 \mathrm{~s}$ of the audio signal. The input signal is sampled and processed at the sampling frequency of $f_{s}=44.1 \mathrm{kHz}$ and quantized with 16 bits per sample. The DSP core C6748 is the main work horse in terms of the digital signal processing and it is responsible namely for the real-time calculation of the de-echo procedure based on Eq. (3). The MCU ARM core is, besides other system oriented tasks, responsible mainly for a regular evaluation and update of the value of the echo lag index $d$ and of the attenuation value $\tilde{A}$. Both values are continuously updated once every two seconds. For a comfortable SW development, we use the floating point arithmetics. The DSP core is exploited by $33 \%$ i terms of available machine cycles. Although the ARM MCU core is not specialized in DSP operations, it is loaded by $24 \%$ only. It indicates that there is a sufficient margin in computational power in both cores available for further improvements and modifications of the implementation, e.g. in terms of a longer echo lag and/or of higher sampling frequency. 


\section{Neural Network World 3/2016, 305-315}

\section{Conclusions}

In this paper we have presented a simple procedure for a single channel echo attenuation. It is based on the evaluation of an autocorrelation sequence of the echo-disturbed signal and on a consecutive filtering of the signal using a comb FIR filter. The proposed method involves no external reference signal. An efficient real time implementation of the presented method on digital signal processor was demonstrated.

\section{References}

[1] AHMAD S., AHMAD T. Implementation of Recursive Least Squares (RLS) Adaptive Filter for Noise Cancellation. International Journal of Scientific Engineering and Technology. 1(4), 2012, pp. 46-48.

[2] BERSHAD N.J., LINEBARGER D., McLAUGHLIN S. A Stochastic Analysis of the Affine Projection Algorithm for Gaussian Autoregressive Inputs. In: IEEE International Conference on Acoustics, Speech, and Signal Processing. USA, 2001, 6, pp. 3837-3840, doi: 10. 1109/ICASSP. 2001.940680.

[3] HANSLER E., SCHMIDT G. Single-Channel Acoustic Echo Cancellation. In: J. Benesty, Y. Huang (eds.), Adaptive Signal Processing. Springer, Berlin, 2003, pp. 59-93, doi: 10. 1007/978-3-662-11028-7_3.

[4] KHOUBROUY S.A., PANAHI I.M.S. Criteria for Estimating an FIR Filter for Cancelling the Feedback Path Signal in Hearing Aid System. Signal Processing. 100, 2014, pp. 101-111, doi: $10.1016 /$ j.sigpro.2014.01.016.

[5] MAHBUB U., FATTAH S.A. Gradient Based Adaptive Filter Algorithm for Single Channel Acoustic Echo Cancellation in Noise, In: 7th International Conference on Electrical and Computer Engineering, Dhaka, Bangladesh. 2012, pp. 880-883, doi: 10.1109/ICECE.2012. 6471691.

[6] MAHBUB U., FATTAH S.A. A Single-Channel Acoustic Echo Cancellation Scheme Using Gradient-Based Adaptive Filtering. Circuits and Systems Signal Processing. 32(5), 2014, pp. 1541-1572, doi: 10.1007/s00034-013-9715-z.

[7] RUPP M. A Family of Adaptive Filter Algorithms with Decorrelating Properties. In: IEEE Transactions on Signal Processing. 46(3), 1998, pp. 771-775, doi: 10.1109/78.661344.

[8] SCALART P., BOUTEILlE F. On Integrating Speech Coding Functions into Echo Cancelling Filters with Decorrelating Properties. In: IEEE International Conference on Acoustics, Speech, and Signal Processing (ICASSP 2002). 2, pp. 1905-1908, doi: 10.1109/ICASSP. 2002.5745000 .

[9] SONDHI M.M., MORGAN D.R. Echo Cancellation for Speech Signals, Wiley Encyclopedia of Electrical and Electronics Engineering. John Wiley \& Sons, Inc., 1999, doi: 10.1002/ $047134608 \mathrm{X}$. W6703.

[10] SVÍTEK M. Investigation to Heisenberg's Uncertainty Limit. Neural Network World. 8(6), 2008, pp. 489-498.

[11] SVÍTEK M. Quasi-Non-Ergodic Probabilistic Systems and Wave Probabilistic Functions. Neural Network World. 9(3), 2009, pp. 307-320.

[12] WATERSCHOOT T.V., MOONEN M. Fifty Years of Acoustic Feedback Control: State of the Art and Future Challenges. In: Proc. of the IEEE. 99, 2011, pp. 288-327, doi: 10.1109/ JPROC. 2010.2090998.

[13] ZHANG W., BENESTY J., CHEN J. Single-channel noise reduction via semi-orthogonal transformations and reduced-rank filtering. Speech Communication. 78, 2016, pp. 73-83, doi: $10.1016 / \mathrm{j}$.specom.2015.12.007.

[14] Development Kit (LCDK), oMAP-L138 [online], [accessed 2016-06-15]. Available from: http://www.ti.com/tool/tmdxlcdk138 
Zahradnik P. et al.: A single channel echo attenuation in signals

[15] C6000 DSP+ARM Processor, oMAP-L138 [online], [accessed 2016-06-15]. Available from: http://www.ti.com/product/omap-1138 